INPLASY

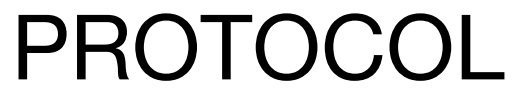

To cite: Gu et al. Effects of Low-FODMAP diet on irritable bowel symptoms in patients with quiescent inflammatory bowel disease: A

SystematicReview and Metaanalysis. Inplasy protocol 202210081. doi:

10.37766/inplasy2022.1.0081

Received: 16 January 2022

Published: 16 January 2022

Corresponding author:

Baijian Gu

jlzygbj@163.com

Author Affiliation:

Changchun University of

Chinese Medicine.

Support: Natural Science

Foundation of.

Review Stage at time of this submission: The review has not yet started.

Conflicts of interest:

None declared.

\section{Effects of Low-FODMAP diet on irritable bowel symptoms in patients with quiescent inflammatory bowel disease: A SystematicReview and Meta-analysis}

Gu, B1; Zhou, J2; Yu, Z3; Shi, C4; Chen, B55.

Review question / Objective: The purpose of this study was to investigate the efficacy difference between low-FOdmap diet and normal diet in the treatment of quiescent inflammatory bowel disease, and the included research method was RCT clinical trial. Fecal calprotectin and quality of life scale were used as the main outcome indicators to compare and analyze the two diets.

Condition being studied: The supporting institution of this study has the right to use CNKI, Cochrane and other databases to search relevant literatures. Most of the participants were front-line clinicians or data statisticians, with professional knowledge of IBD and data analysis ability.

INPLASY registration number: This protocol was registered with the International Platform of Registered Systematic Review and Meta-Analysis Protocols (INPLASY) on 16 January 2022 and was last updated on 16 January 2022 (registration number INPLASY202210081).

\section{INTRODUCTION}

Review question / Objective: The purpose of this study was to investigate the efficacy difference between low-FOdmap diet and normal diet in the treatment of quiescent inflammatory bowel disease, and the included research method was RCT clinical trial. Fecal calprotectin and quality of life scale were used as the main outcome indicators to compare and analyze the two diets.

Condition being studied: The supporting institution of this study has the right to use CNKI, Cochrane and other databases to search relevant literatures. Most of the participants were front-line clinicians or 
data statisticians, with professional knowledge of IBD and data analysis ability.

\section{METHODS}

Participant or population: patients with quiescent inflammatory bowel disease.

Intervention: The Low-FODMAP diet.

Comparator: Normal diet.

Study designs to be included: Randomized controlled trial.

Eligibility criteria: Inflammatory bowel disease diagnosed according to the standards of the American Gastrointestinal Association or the European Society for Gastrointestinal Endoscopy.

Information sources: From inception up to December 20, 2021, the PubMed, Web of Science, Embase, AMED,Cochrane Library, CNKI, VIP, CBM, and Wanfang databases will besearched.

Main outcome(s): The primary outcome measures will include the Harvey Bradshaw Index or MAYO score, and Fecal calprotectin.

Quality assessment / Risk of bias analysis: STATA software was used for sensitivity analysis, and the sensitivity of the literature was corrected by the change of effect size after deleting one of the literature.

Strategy of data synthesis: STATA software was used for registry analysis, and I squared $>50 \%$ and $P<0.1$ were considered as heterogeneity, heterogeneity selection random effect combined effect size, and heterogeneity selection fixed effect combined effect size.

Subgroup analysis: A subgroup analysis will be performed for ulcerative colitis and Crohn's diseaseNo subgroup analysis will be performed.

Sensitivity analysis: STATA software was used for sensitivity analysis, and the sensitivity of the literature was corrected by the change of effect size after deleting one of the literature.

Language: No language limits in the study.

Country(ies) involved: China.

Keywords: FODMAP diet.

Contributions of each author:

Author 1 - Baijian Gu.

Email: jlzygbj@163.com

Author 2 - Jianhua Zhou.

Email: 645661949@qq.com

Author 3 - Zhe Yu.

Author 4 - Chong Shi.

Author 5 - Bixin Chen. 